Nuevos Modelos Locales de Relaciones Laborales en Italia. Una Primera Reflexión.

FRANCESCA MALZANI*

Iberoamerican Journal of Industrial Relations

ISSN 2173-6812

VOL. 34, (2016)

pp. 13-24

Dy puhues 


\section{FECHA RECEPCIÓN:}

17/08/2016

FECHA REVISIÓN:

$17 / 08 / 2016$

\section{Palabras clave}

Negociación descentralizada; Bienestar; Conciliación laboral.

\section{Resumen}

El trabajo identifica algunas tendencias de la negociación colectiva en Italia, en un contexto nacional de cambio profundo en la relaciones laborales.

En particular, después de describir el marcentra en la ado mentos de welfare y conciliación en la negociación de segundo nivel en la Provincia de Brescia.

La elección de estas dos cuestiones no es casual: son aspectos que, junto con otros por supuesto (por ejemplo la formación, la participación, etcétera) contribuyen a la construcción de modelos holísticos de calidad del trabajo.

\section{FECHA ACEPTACIÓN:}

17/08/2016

FECHA PUBLICACIÓN:

$17 / 08 / 2016$

\section{KeYWORDS}

Decentralized bargaining; welfare; conciliation.

\section{ABSTRACT}

The aim of this paper is to identify some trends of collective bargaining in Italy, in a context of industrial relations profoundly changed in recent years.

In particular, moving from the changed landscape of industrial relations, the paper focuses on the adoption of instruments of welfare and conciliation in the second level agreements in our area.

The choice of these two issues is not accidental: it falls under the aspects that, joined with others of course (eg. training, participation, dels of quality of work 


\section{Introducción.}

En 2005, Pietro Ichino publicó una obra con el título provocador $A$ che cosa serve il sindacato? (Ichino, 2005).

La monografía se centra en la investigación sobre la capacidad del sindicato de presentar se como interlocutor estable en los procesos de profunda transformación de las estructuras de organización, es decir, en la transición desde los modelos tradicionales de fordismo/taylorismo hacia modelos de lean production, world class manifacturing (en general, sobre el cambio del papel del sindicato en los últimos años, Visser, 2013).

Pietro Ichino criticó al sindicato italiano, y en particular a Fiom (Federación de Empleados y Obreros Metalúrgicos - Cgil), por permanecer anclada en el convenio colectivo nacional y, por ello, no tener en cuenta las características de cada empresa, especialmente para aquellas insertas en la dinámica de la competitividad global. Segun el autor, el enfoque en la negociación a nivel central no permitía mejorar la actividad de las empresas través de modelos de negociación más cooperativos y menos jerárquicos, introduciendo sistemas de clasificación profesional más ágiles, salarios vinculados a la productividad y herramientas de promoción de la participación directa de los trabajadores (Berta, 2014).

En un artículo reciente, Gian Primo Cella ha vuelto a preguntarse cuál es el futuro de la negociación colectiva, desplazando la atención sobre el resultado de la negociación (el convenio mismo) y las trayectorias tomadas por las relaciones laborales (Cella, 2016) en un sistema de capitalismo "avanzado" (Baccaro, Howell, 2011). La crisis económica ha afectado a la balanza comercial y a las estrategias de negociación: ha fallado la unidad sindical, proliferan acuerdos "separados", acuerdos que establecen excepciónes a los de nivel superior y, a menudo, en Italia se asigna a la negociación de segundo nive la busqueda de exit strategy (in pejus) respecto a las normas establecidas por lo convenios nacionales más que el relanzamiento de la competitividad territorial y de la empresa (Guarriello, 2016). La impresión, segun parte de la doctrina, es la de un tránsito hacia una trayectoria común "neoliberal" (Bologna, 2016).
En los ultimos años, se ha hablado así de "erosión", "disminución”, "desaparición" de las relaciones laborales, tal como eran conocidas en el siglo XX (Carrieri, Treu, 2013, 7), orientada a una regulación con rasgos universales y una dimensión nacional, para dejar espacio a nuevas realidades y convergencias, sea tanto en el ámbito supranacional/sectorial como en el local/ de empresa. Este proceso esta relacionado a lo que occurrió con la competencia nel mercado global y a las nuevas formas del capitalismo moderno; como afirmó Streek, hablando de Alemania pero con un asunto que puede extenderse a otras experiencias, "fragmented reorganization of cooperative relations between capital and labor at the level of the firm replaces an obligatory social order with voluntarily contracted, individualized arrangements, responding to and controlled by competitive markets" (Streek, 2009, p. 86).

Sin duda un ejemplo paradismático de tal desplazamiento del eje de la negociación colectiva tuvo lugar en el grupo Fiat en el período comprendido entre 2010-2012.

La ruptura de las negociaciones con Fiom, el abandono del sistema confederal (convenio colectivo sectorial) y la profundización de la división entre los diferentes sindicatos, van a ofrecer un escenario para reflexionar en torno al papel del sindicato, la futura organización de las relaciones laborales y las perspectivas de modelos "locales" de diálogo social.

Aunque generalmente se acepta que este abandono del convenio sectorial estatal por parte de la Fiat no produjo el efecto perturbador temido, sin embargo es cierto que derivó en la aparición de nuevas tendencias en la estructura de la negociación colectiva y en las relaciones entre los actores sociales.

En particular, el caso Fiat implicó una valorización del convenio a nivel de empresa y, más en general, la doctrina se ha planteado los desafíos y efectos que pueden alcanzar los convenios a nivel descentralizado.

El objetivo de este trabajo es intentar entender - a través del material analizado por el Observatorio sobre la negociación colectiva del Departamento de Derecho de la Universidad de Brescia - cómo está evolucionando la estructura de la negociación colectiva y comprobar si, en la experiencia de nuestra Provincia, los convenios de ámbito descentralizado (de empresa o locales) han logrado transmitir buenas prácticas y promover el desarrollo local desde una perspectiva de "solidaridad productiva" (De Felice, 2015, 83), superando la idea de que la descentralización de la negociación colectiva es sinónimo de mera derogación de lo dispuesto en el convenio nacional y de "corporativización / individualización de la legislación laboral y sindical” (Zoppoli, 2015, 47; Vaughan-Whitehead Daniel, 2015, 28 ss.).

Antes de abordar este debate hay que presentar, aunque sea brevemente, el marco normativo y los antecedentes de hecho sobre los que se asienta la necesidad de estudiar como este asunto ha ido madurado. Imposible hablar de descentralización y trazar nuevas perspectivas sin hablar del caso Fiat. 


\section{El caso Fiat.}

La conducta de las partes en el caso Fiat se construye a través de lecturas diametralmente opuestas: por un lado, la compañía atribuye a la Fiom la ruptura de las negociaciones (Rebaudengo, 2015); por otro lado, la doctrina (Aa.Vv., 2011) y la opinión pública, con presencia de la prensa, han enfatizado una actitud de rechazo respecto de la Fiat y un punto de inflexión sin precedentes en las relaciones laborales hacia, como ya he mencionado, la salida del sistema de negociación colectiva nacional confederal y las consecuencias de esa elección.

La historia comenzó en la sede de Pomigliano, en el 2010, con la reorganización de las relaciones laborales en una fábrica considerada ineficiente: nació una nueva empresa llamada Newco y se adoptó en ese ámbito un nuevo convenio colectivo.

Se trata de un convenio colectivo específico de primer nivel (CCSL), el único aplicable en este ámbito, firmado por Fiat para todas las empresas del grupo. Al mismo tiempo, tuvo lugar la salida definitiva de Fiat de Confindustria (la gran asociación empresarial de la industria italiana) al inicio de 2012 y la rescisión para la empresa de los convenios colectivos sectoriales, ante el rechazo del Acuerdo de 1993 sobre la representación sindical unitaria (r.s.u.).

Uno de los puntos controvertidos del acuerdo surgía en relación a las cláusulas de exigibilidad/cláusulas de responsabilidad ${ }^{1}$, dirigidas a garantizar el cumplimiento de las disposiciones contractuales. Tales cláusulas recuerdan la tradición de los sindicatos anglosajones y la experiencia de América del Norte, donde está clara - especialmente en los acontecimiento más recientes (incluyendo, los relativos a la Chrysler y Fiat en 2008-2009) - la predisposición a la cooperación con los empresarios para hacer frente a la crisis.

Sin embargo, dada nuestra experiencia sindical (Liso, Corazza, 2015), son cláusulas difíciles de aplicar debido a un modelo de pluralismo sindical que sigue estando orientado a un enfoque antagónico frente a la contraparte empresarial, aunque hoy en día muy lejos del "conflicto permanente" de los años Setenta.

De hecho, aunque este tipo de cláusulas a menudo se incorporan en los más recientes acuerdos locales, no se hace referencia a las sanciones, lo que frustra la ratio de la reciprocidad vinculante.

En este contexto ha tenido lugar la división entre la empresa y los sindicatos: en particular, la Fiom no firmó el nuevo convenio de ámbito empresarial, a pesar del resultado

1. Un ejemplo lo ofrecen tradicionalmente las cláusulas de paz sindical, ya utilizado en el pasado por los interlocutores sociales. En el Acuerdo de Fiat la empresa se reserva el derecho de no pagar las cuotas sindicales, no reconocer permisos sindicales y proceder a la imposición de sanciones disciplinarias incluido el despido, si no se hubiera respetado el acuerdo. positivo del referéndum (la mayoría de los trabajadores estaban a favor, en parte porque de lo contrario se procedería al cierre de la fábrica, pero pese a ello con numerosos desacuerdos). Lo mismo ocurrió en la fábrica de Mirafiori.

El hecho de no firmar el acuerdo provoco que la empresa interpretase que la Fiom a partir de entonces no podía contar con representantes sindicales a nivel de empresa y el acceso a los derechos del título III de Statuto dei lavoratori relativos a la acción sindical en la empresa. La batalla legal culminó con la decisión del Tribunal Constitucional de julio 2013, n. 231, a la que volveré en el § 2.1.

En términos de relaciones individuales de trabajo la demanda se refiere a la ausencia de readmisión en Pomigliano de 19 trabajadores que pertenecen a Fiom. Aunque no será abordado tal asunto por este estudio, es interesante señalar el razonamiento dado por el juez, que se caracteriza por la tutela que ofrece el derecho antidiscriminatorio ${ }^{2}$

El Tribunal de Apelación reconoció la existencia de la discriminación por razón de creencia - como expresión de la libertad ideológica que se refiere a la persona, fundándose en la interpretación ofrecida por el TEDH (Tribunal Europeo de Derechos Humanos) (Barbera, Protopapa, 2014) - condenando la empresa a la reincorporación de un número de trabajadores que garantice el mantenimiento del porcentaje de afiliados a la Fiom entre los trabajadores de la empresa.

\subsection{El caso judicial: los representantes sindicales en la empresa.}

El rechazo de la Fiom a firmar el CCSL en Pomigliano y Mirafiori dio lugar, de conformidad con el art. 19 de Statuto dei lavoratori, a la negación por parte de la empresa del derecho a tener representantes sindicales en la empresa misma.

Ante esta negativa, la Fiom apeló en conformidad con el art. 28 de Statuto dei lavoratori que se refiere al procedimiento relativo a la represión de conductas antisindicales. Los Tribunales de Modena (orden 4.6.2012), Vercelli (orden 25.9.2012) y Torino (orden 12.12.2012) han planteado la cuestión de la constitucionalidad de la norma ex art. 19.1.b Statuto dei lavoratori), con fundamento en los artículos 2, 3 y 39 de la Constitución por las siguientes razones: a) la firma del convenio no es expresión de un consenso real de los trabajadores; b) el requisito de la firma afecta a la libertad de elección en el ejercicio de la acción sindical, porque según la norma legal ordinaria sólo la firma permite el acceso a los derechos del título III de Statuto dei lavoratori; c) se produce una desigualdad de trato entre las asociaciones que tengan igual fuerza representativa en la empresa.

2. Tribunal Roma, 21.6.2012, en Foro It., 2013, I, 1674; Tribunal de Apelación Roma 9.10.2012. 
El Tribunal Constitucional, en julio de 2013, dictó una sentencia de constitucionalidad pero condicionada a la interpretación de la norma legal, sentencia interpretativa (pronuncia addittiva), sancionando a la postre la no adecuación a los parámetros constitucionales del articulo 19 en la medida en que no prevé que la representación sindical en la empresa pueda constituirse también en el marco de las asociaciones que no han firmado el acuerdo, pero han participado en la fase de negociación, considerando a este requisito como una forma de representatividad exhaustiva (Napoli, 2014: 36 ss., habla de "capacidad máximo de la sentencia").

El sindicato, a diferencia de los actores políticos, no ejerce como un representante que se limita a ser (standing for), sino que se centra en el hacer (acting for), que se manifiesta en la negociación colectiva (Cella, 2013: 513 ss.), con diferente grado de autonomía a nivel naciona o de empresa, debido tambien a la diversidad y variedad (range) de posiciones en las plataformas de negociación.

La propuesta de Fiat en Pomigliano y Mirafiori apareció casi "blindada", justificado por una necesidad imperiosa de aumentar la eficiencia ante la perspectiva de la realización de inversiones significativas (o el inminente cierre y traslado de las mismas instalaciones).

\section{¿Qué tipo de efectos asume esta dicotomía en el juicio del Tribunal Constitucional?}

La literalidad del art. 19 de Statuto dei lavoratori es clara en esta materia y traza una línea de demarcación entre la posición de los firmantes y los no firmantes del convenio colectivo en quanto a su posibilidad o no de constituir representaciones en la empresa. Sin embargo, lo que no es tan claro, como afirma Cella, es la distinción entre el actuar (por un fin negocia descentralizado) y el ser (para la aplicación del acuerdo, la legislación laboral, los derechos de información, etc.). Los dos perfiles se entrelazan y la "representatividad efectiva" (la firma del acuerdo) transmitida por el texto post-referéndum de 1995 se reinterpreta a la luz de una fenomenología de relaciones y conductas muy diversas (ausencia durante las negociaciones, presencia discontinua, obstruccionismo, colaboración, etc.) que puede o no dar lugar a la firma del convenio. El proceso y el resultado final pueden no converger (Del Punta, 2013: 534), pero la negación de los derechos sindicales a los que "actuaron en virtud de su representatividad" conduce a una paradoja inaceptable.

Este resultado contradice la premisa del criterio de selección subyacente en el art. 19 de Statuto dei lavoratori, traicionando los propósitos promocionales de esa regla, y conduce a una violación del artículo 39 de la Constitución (libertad de asociación), así como de los artículos 2 y 3 , expresiones del principio de solidaridad y del principio de igualdad, que derivan de la Constitución.

Esta contradicción es aún más chocante en el nuevo contexto de las relaciones laborales, en el que la unidad de los sindicatos se ha convertido en la excepción y no en la regla, como demuestra la experiencia de los acuerdos separados, es decir los firmados por algunos sindicatos pero no por todos ellos.
El Tribunal, por lo tanto, en este caso, hace una evaluación doble ${ }^{3}$ : cree, por una parte, en la participación en la negociación como un índice de representatividad y, por otra, en el rechazo a la firma como una expresión de la libertad de asociación.

El disenso debe ser garantizado y no edulcorado, como sucedería si se confirmara la lectura ofrecida por Fiat. No se puede aceptar que los conflictos en las negociaciones se traduzcan en una farsa que con el tiempo daría lugar, de todos modos, a la firma del convenio con el fin de no excluirse del acceso a los derechos sindicales.

Denegar el reconocimiento de los derechos sindicales sería, por lo tanto, un ataque a "los valores del pluralismo y de la libertad de los sindicatos», favoreciendo el comportamiento oportunista del empleador: de esta forma el mismo empleador podría sutraerse de la firma del convenio y no admitir representantes sindicales en la empresa (Leccese, 2013: 545 ss.; habla de «una forma impropia de castigo del disentimiento», p. 550) o acreditar solo a representantes complacientes.

El art. 19 es, por tanto, inconstitucional en la medida en que no prevé que la representación sindical de empresa (r.s.a.) pueda constituirse por los sindicatos que, aunque no firmantes del convenio colectivo aplicable en la unidad de producción, participaron en la negociación como representantes de los trabajadores de la empresa, superando así el obstáculo que resultaría de la ausencia tout cout de convenio colectivo o el fracaso en la firma del mismo convenio.

Despues de la sentencia del Tribunal Constitucional, Fiat emitió un comunicado, por el que dio la bienvenida al pronunciamiento judicial, y reconoció el derecho de Fiom a elegir representantes en la empresa.

Hay otro asunto igualmente mui controvertido Desde hace mucho tiempo hay discusiones sobre los criterios a través de los cuales se debe medir la representatividad sindical dejando abiertas este debate toda una serie de cuestiones. Ante todo, la pregunta es si necesitamos una intervención legislativa, tal cómo ha ocurrido en la negociación de empleo público; cómo continuar garantizando la admisión a la mesa de negociaciones; si establecer una obligación de negociar; cómo regular la conciliación entre la actividad negociadora y los acuerdos "separados", etc.

El Testo Unico sulla Rappresentanza de 10 enero 2014 (Carinci, 2014) - no suscrito por parte de las empresas del Grupo Fiat - identifica un umbral de admisión a la mesa de negociaciones por la estipulación el convenio nacional (5\%) calculado sobre el número de miembros (atribuido a través de las cuotas sindicales pagadas por los trabajadores) y los datos electorales (votos obtenidos en las elecciones de r.s.u.). El Acuerdo Interconfedera de 26 noviembre 2015, entre los principales sindicatos nacionales (Conf-commercio, Cgil,

3. El Tribunal no consideró que hubiera margen para una interpretación constitucional y ha reconocido la inconstitucionalidad de la norma. 
Cisl e Uil) amplía la gama de criterios útiles para medir la representatividad sindical y, por lo tanto, para determinar el acceso a la mesa de negociación para aquellos que llegan a umbral del $5 \%$. El acuerdo, de hecho, repite el criterio del número de representaciones para la deducción de la cuota de afiliación sindical y el consenso conseguido en la elección de representantes, pero a ellos se suman dos más: a) el número de conflictos individuales, plurales y colectivos, en su sector de aplicación, en los tres años antecedente e inicio de negociaciones para la renovación de convenio colectivo nacionl de trabajo (Ccnl); b) el número de prácticas para el desempleo certificable por parte del servicio público.

La imagen resultante de las páginas anteriores, con la peculiaridad del caso Fiat, es reveladora de un sindicalismo confederal en crisis.

Sin embargo, esto no se ha traducido en una involución de la negociación, sino en un desplazamiento del eje de las negociaciones hacia la "periferia" negocial (el territorio local la empresa).

\section{Modelos locales de negociación.}

La literatura sociológica nos propone la imagen no de una reducción de las actividades de los actores sociales, sino de un cambio hacia la descentralización.

Este ámbito parece permitir comprobar de soluciones innovadoras, por la proximidad a problemas y realidades con una especificidad no reproducible en un ámbito superior y porque ofrece una mayor libertad de movimiento y de participación de las instituciones locales.

Parece, también, fomentar en Italia formas de concertación social, desde hace mu cho tiempo abandonadas, o simplemente desarroladas a nivel nacional (Regalia, 2015a 3 ss.), que encuentran a otro ámbito una nueva oportunidad gracias a la movilización de abajo hacia arriba (bottom up)

La doctrina proporciona una clasificación de estas diversas formas, que van desde la negociación hasta la concertación social, dando la sensación de movilización a nivel de empresa o territorial: a) contrattazione territoriale di settore o comparto (segundo nivel de negociación colectiva en sectores específicos como el artesanal, la construcción, el comercio, el turismo, que a menudo constituyen los distritos productivos, etc.); b) contrattazione territoriale cross-settoriale (convenios colectivos relativos a una zona geográfica determinada); c) patti territoriali e iniziative multilaterali (pactos territoriales orientados al desarrollo local y al crecimiento del empleo); d) negoziazione o concertazione sociale (destinada a la promoción de la política social y welfare).

Con respecto a la concertación como técnica de governance del territorio (Regalia 2015b, 102 ss.) hay un cierto activismo (por ejemplo, pactos territoriales para el empleo que pueden negociarse en el marco de los programas de iniciativa nacional o europeo).
Esto ocurre, de este modo, en contraste con la reducción progresiva del método a nivel central después de la gran era de los años Noventa.

El lanzamiento de la programación negociada, expresión del partenariado socio-económico de un área determinada con la finalidad de conseguir el desarrollo local, habia elegido el nivel local como lugar privilegiado para promover el desarrollo y el empleo (il Patto per il lavoro de 1996, il Patto per lo sviluppo e l'occupazione de 1998), pero el entusiasmo inicial no consiguió un uso efectivo de las herramientas (pactos territoriales, acuerdos de área): los temas críticos y los obstáculos para el despegue del sistema estaban relacionados con la ineficiencia del gobierno/instituciones locales, la renuencia de las empresas y los sindicatos a superar conflictos ideológicos, así como la falta de legislación de apoyo a la negociación (Zoppoli, 2015, 34 ss.; De Felice, 2015, 92, con especial atención a las Regiones del sur de Italia).

En la perspectiva del ordenamento intersindical, los Acuerdos de 2009, 2011, 2013 y 2014 , en la definición de la estructura de la negociación colectiva, se acercaron al nivel loca de una manera "ondulante": el de 2009 asigna a la negociación local márgenes de ajuste in peius, para hacer frente a la crisis o para lograr un aumento del empleo; el de 2011 borra la referencia expresa a ese nivel de la negociación - se refiere en sus premisas al nivel descentralizado y luego a la negociación a nivel de empresa - que vuelve de la mano del art. 8 de la Ley n. 148/2011 (contratti di prossimità); ; el de 2014 confirma la elección semántica del 2011 (Zoppoli, 2015, 36 ss.) ${ }^{5}$.

En cualquier caso, los acuerdos citados asignan al nivel descentralizado de negociación la tarea de mejorar la competitividad de las empresas, proponiendo "cláusulas de salida" con respecto a lo regulado por el convenio colectivo nacional; tendencia que se repite en muchos países, incluido en Francia, España y Alemania, especialmente con respecto a los salarios a través de cláusulas variadas (opening clauses, hardship clauses, opt-out clauses, inability-to-pay clauses: Keune, 2011, p. 87 ss.). En general, las relaciones laborales están

4. Se trata de acuerdos que, de conformidad con el art. 8 de la Ley n. 148/2011, tienen alcance de derogación de las disposiciones contenidas en los convenios colectivos y disciplina legal si se ha suscrito sobre la base de un voto por mayoría de las asociaciones más representativas en el ámbito nacional o local o de sus sindicatos que operan en la empresa, destinada a obtener empleo más elevada, calidad de los contratos de trabajo, adopción de formas de participación de los trabajadores, aumento de la competitividad y de los salarios, gestión de las crisis empresariales, inversión y puesta en marcha de nuevas actividades. Los temas tratados son indicados por e legislador, pero el espectro es bastante amplio (clasificación de personal, equipos audiovisuales, nuevas tecnologías, contratos a tiempo determinado, etc.).

5. En el Acuerdo Interconfederale separado (sin la Cgil) en noviembre de 2012 (Linee programmatiche per la crescita della produttività e della competitività in Italia), se valoraba el ámbito territorial ofreciendo la oportunidad de ajustar los perfiles relacionados con de-contribución y desgravación fiscal de salario de productividad, especialmente por las pequeñas empresas que no cuentan con una negociación de empresa. 
luchando por encontrar una estrategia reguladora creíble para abordar la desigualdad en el mercado del trabajo (Ackers, 2016).

En paralelo, y como bien destaca la doctrina (Zoppoli, 2015), el sistema estatal ofrece elementos para apoyar la negociación y la concertación territorial local, en algunos casos con la puesta a disposición de recursos públicos: contratti di riallineamento; contratti d'area; programación negociada; contratti di prossimità con eficacia erga omnes; acuerdos territoriales para destinar cuotas de salario al incremento de la productividad asociadas a la reducción de impuestos y de las contribuciones.

El dinamismo de la actividad negocial, aunque a un nivel diferente, por lo tanto, confirma la vitalidad del ordenamiento intersindical. Por eso en el reciente Acuerdo entre Cgil, Cisl y Uil del 14 enero 2016 (Un moderno sistema di relazioni industriali. Per un modello di sviluppo fondato sullinnovazione e la qualità del lavoro) se valoran mas tipologías de negociación descentrada (aziendale, territoriale, distretto, sito, filiera), y a esas se asigna la tarea de impulsar la competitividad y el desarrollo económico y social.

Más allá de la clasificación, que se ha mencionado brevemente en este párrafo y que se detiene en los aspectos menos claros descritos en literatura, el objetivo de este trabajo es identificar la tendencia diseñada por los interlocutores sociales en la Provincia de Brescia.

Las preguntas a las que trataré de dar respuesta son el Quien (es decir, qué sindicatos se encuentran incolucrados) y, sobre todo, el Como (el tipo de acuerdo, el número, la posición en el tiempo y próxima a la crisis, la estipulación unitaria o separada) la negociación de segundo nivel es capaz de transmitir soluciones virtuosas y, al mismo tiempo, útiles a las necesidades de la empresas y del territorio.

\section{Los convenios censados por el Observatorio de la negociación colectiva en la Universidad de Brescia (Osmer): tendencias de la negociación del segundo nivel en la Provincia de Brescia.}

La Provincia de Brescia es la segunda provincia más industrializada de Lombardía después de Milán. El entorno productivo se compone principalmente de pequeñas y medianas empresas, además de los que hay, sin embargo, empresas industriales importante sobre todo en el sector del hierro y acero.

El Observatorio, enraizado en la Universidad, nació de una necesidad muy sentida en el territorio: estudiar las relaciones laborales y las tendencias en la negociación colectiva en ámbito local. Al Observatorio se adhieren los principales sindicatos de trabajadores (Cgil, Cisl e Uil), de empresarios (Aib: Associazione industriale bresciana), profesionales de asesoria en materia de trabajo (Consulenti del lavoro) y la Dirección Territorial de Trabajo (articulación periférica del Ministerio de Trabajo).

El Observatorio al final de octubre 2015 ha recogido y supervisado 1186 convenios por el periodo $2008-2013,400$ por el año 2014 , y se mantiene trabajando en la catalogación de los convenios de 2015 y 2016

El Monitoreo, en los últimos años, se ve facilitado por el requisito de la presentación a la Dirección Territorial del Trabajo de convenios que se benefician de la reducción del impuesto/de-contribución prevista para el salario de productividad ${ }^{6}$. La obligación fue confirmada por la Ley del Empleo (Jobs Act), que prevé el depósito, en forma electrónica, de los convenios que tratan acerca de salario de productividad y la puesta a disposición de las autoridades competentes (art. 14, decreto n. 151/2015).

La mayoría de los acuerdos se refieren a la industria y a los servicios, seguidos por el crédito, el comercio, la artesanía, la construcción y la agricultura: esta distribución refleja las características productivas de la Provincia de Brescia.

Para el período 2008-2013, el 97\% de los convenios tiene naturaleza integradora/ normativa y el porcentaje restante lo integra los acuerdos de gestión (accordi gestionali); la mayoría de los convenios fueron firmados por las r.s.u. (aproximadamente 73\%) con la presencia de los tres sindicatos principales (Cgil, Cisl, Uil).

Los temas identificados para construir la base de datos, y así obtener la catalogación, son los siguientes (muchos están presentes conjuntamente en un mismo acuerdo): reclutamiento, movilidad interna, formación, riesgos laborales, representante de seguridad y salud, información y formación, consulta, tiempo de trabajo, horario flexible, despido individual, despidos colectivos, redes de seguridad social, subcontratación, transmisión de empresas, contratos atípicos, relaciones laborales, comités conjuntos, igualdad y discriminación, bienestar, conciliación e igualdad de oportunidades, premio variables/ productividad $u$ otros premios ${ }^{7}$.

En muchos casos, las negociaciones se han centrado en los acuerdos de concesión (concession barganing), en consonancia con la situación de crisis económica que comenzó en 2008 y que aún no se ha completado.

De acuerdo con la doctrina, hay una conexión llamativa entre la reducción de los costes

6. Ley n. 228/2012 y decreto 22 enero 2013

7. La mayoría de los acuerdos se refiere a los premios de productividad y la composición de los elementos para el cálculo de la reducción progresiva de la cuota fija. Para el período más reciente, el aumento de los acuerdos en este perfil también se justifica por el hecho de que la Ley de Estabilidad de 2013 (Ley n. 228/2012) introdujo una especial desgravación fiscal del salario para aumentar la productividad (remuneración de la productividad) y esta medida se continuó en los años siguientes. 
laborales o un aumento significativo (hasta $100 \%$ ) del componente variable del salario de productividad o un horario de trabajo más flexible (aumentar el número de turnos por semana, ciclo continuo, uso de horas extras, reducción de los descansos, etc.) frente a una mayo seguridad en el empleo, incluso a través de las inversiones en las fábricas, relanzamiento productivo y reentrenamiento del personal (Tomassetti, 2015).

Me decidí a centrar la atención en los convenios que tengan en cuenta, en particular la adopción de instrumentos de welfare corporativo y de conciliación. La elección no es casual: son aspectos que, por supuesto junto con otros (por ejemplo la formación, la participación, etcétera), contribuyen a la construcción de modelos holísticos de calidad de trabajo. Parece, de hecho, existir una circularidad entre los dos temas, la cual es evidente a partir del examen de las herramientas utilizadas para construir paquetes de servicios que se pueden colocar en el welfare corporativo (un ejemplo son los voucher por servicios personales y de la familia, los acuerdos con guarderías, etc.) (Di Nardo, 2015).

Estas cuestiones también fueron objeto de «negociación social territorial» (D'Arcangelo, 2012, 143 ss.), promovida por la Cgil nacional a partir de 2009, que propone una dimensión más amplia de la intervención (municipal, provincial) en comparación con el nivel de empresa, que todavía es un buen punto de partida para la experimentación de soluciones innovadoras. La participación de los interlocutores sociales, las instituciones y los particulares, especialmente en las zonas con mayor riesgo de exclusión social, parece avanzar en esta experiencia hacia formas de concertación social o programación negociada (supra § 3), pero lo que importa destacar es la actitud esquizofrénica hacia la centralidad sostenida de los temas de bienestar y conciliación y la baja adopción de políticas de apoyo al territorio y la familia (Ires, 2015)

Las herramientas de welfare corporativo son una forma importante de recuperación de poder adquisitivo/poder económico de los trabajadores; la conciliación es un objetivo reiterado regularmente en las agendas políticas nacionales, pero con baja tasa de efectividad, que a menudo desembocan en una salida temprana de las mujeres (que llevan las tareas de cuidado de menores y personas de edad avanzada) del mercado de trabajo y un aumento, en el medio y largo plazo, de la pobreza de las familias.

Son perspectivas que forman parte del debate sobre el llamado segundo sistema de bienestar, que interviene en áreas de necesidad, por desgracia cada vez mas intensas, no cubiertas por la intervención pública (Maino, Ferrera, 2013).

La construcción de un lugar de trabajo "de calidad" es un objetivo en el que debe de existir un mayor compromiso, para mejorar no sólo la salud de los trabajadores (en su sentido más amplio, incluyente de bienestar), sino también la productividad y la competitividad de la empresa. Es innegable que existe una relación entre la salud organizaciona y la productividad de la fuerza laboral en términos de reducción de las bajas por enferme- dad, menor rotación, disponibilidad a la asunción de nuevas tareas y en algunos casos a tolerar "sacrificios temporales".

En el período 2008-2014 el porcentaje de acuerdos que se refieren a conciliación y welfare es de aproximadamente un $10 \%$.

Los instrumentos de conciliación asumidos por la empresas son mui variados: promoción de un horario flexible de trabajo, tiempo parcial voluntario (aumento del porcentaje sobre la fuerza laboral en general) y el trabajo a distancia; acceso al tiempo parcial por tareas de cuidado de los familiares con enfermedades graves acreditadas; concesión de licencias no remuneradas por exigencias familiares; flexibilidad horaria en el primer año de vida de un niño para el trabajador solicitante (padre o madre); adaptación del tiempo de trabajo durante las etapas correspondientes al acceso a las guarderías de los menores de 3 años para el trabajador solicitante (padre o madre); formación antes de la licencia de maternidad para la transición de las tareas; formación después del permiso para actualizar la madre trabajadora.

Las herramientas de welfare corporativo van a consistir en: voucher para los trabajadores con ausencias reducidas (ej. menos de 3 días); seguro de salud; contribuciones para los gastos incurridos por los empleados, cónyuge e hijos (guarderías, jardines de infancia, otros niveles escolares, master, cursos de idiomas, campamentos de verano, compra de libros de texto, comedor escolar, fondos de pensiones); aumento de la contribución del empleador al fondo de pensiones complementario; becas para los hijos de los empleados que se han distinguido por sus méritos académicos peculiares (en escuelas secundarias y universidades) ${ }^{8}$; acuerdos con guarderías; activación de jardines de infancia en el lugar de trabajo; voucher de compra.

En los últimos acuerdos la atención a las cuestiones mencionadas anteriormente tienden a disminuir: en 100 acuerdos firmados al inicio de 2015 sólo en 2 existe referencia a

8. Fuera de la provincia de Brescia, en los temas aquí analizados, el contrato de Luxottica, de junio de 2013, dedica un amplio espacio para apoyar la educación de los niños de los empleados (con becas y pago de cuotas escolares), pero también, en una perspectiva de "bienestar territorial", a la inversión en proyectos destinados a la lucha contra el abandono escolar prematuro a través de acuerdos con escuelas locales. En el convenio de Ferrero, de julio de 2014, se destaca el aumento de 2 a 3 medias jornadas de licencia para las visitas pediátricas de los niños entre las edades de 0 y 14 años; un día libre adicional, respecto al convenio colectivo nacional, al padre en el nacimiento del niño y la posibilidad de activar formas de tiempo parcial para necesidades específicas. En abri de 2015, el grupo de Zara ha firmado su primero convenio de empresa y, en términos de conciliación, prevé la reducción de las rondas finales para las madres que trabajan y un permiso sin sueldo tras el permiso parental de 12 meses. 
los instrumentos de conciliación o el bienestar corporativo ${ }^{9}$. En la segunda parte de 2015 aparecen un par de acuerdos que incluyan intervenciones de bienestar corporativo (vou cher de compras, regalo de Navidad).

Casi todos los acuerdos de 2015 estan relacionados con el cálculo del premio de productividad, poniendo el acento en el componente variable aunque no necesariamente desde una óptica de participación en el éxito de la compañía (Ricciardi, 2014, 31 ss.), con el trabajo flexible o a turnos para satisfacer las necesidades de producción. Estas últimas introducen vaivenes, inclinándose así la organización hacia modelos just in time (en la producción tiende por ejemplo a desaparecer el "almacén" porque cuesta mucho). También se encuentran en la segunda mitad de 2015 varios acuerdos sobre la transferencia de empresa o su rama.

Estas acciones, o lineas de tendencia, se colocan en una lógica de reducción de los costes laborales, sin duda útil para las empresas, pero no estimulan la adopción de políticas industriales y la revitalización del territorio

La reducción gradual de la parte fija del salario, establecido a nivel nacional, ha acelerado en los últimos años el proceso de «erosión de la función de la solidaridad del convenio colectivo nacional» (Campanella, 2013, p. 7) que es expresión de la función de composición social desempeñada por la negociación. En abril de 2015 es todavía el grupo Fiat-Chrysler Automobile a llamar la atención con un acuerdo sobre un nuevo «sistema retributivo premiale». El Acuerdo reduce a tres macro-áreas profesionales, en comparación con los 8 niveles de clasificación del Convenio nacional Metalúrgicos, y define, a un solo ámbito de negociación, la construcción de la parte premial de la retribución. Es un bonus calculado sobre el rendimiento anual del establecimiento y sobre la performance en cuatro años en la área geográfica de referencia (la áreas son: Europa, Africa e Medio-oriente; Nord America; Sud America; Asia) (Aa.Vv, 2015).

En los escasos acuerdos formalizados en la Provincia de Brescia en el 2015 dedicados a las cuestiones que aquí nos interesan se encuentran algunas reglas interesantes: horarios de salida flexibles comprimiendo la pausa para el almuerzo; 2-3 días de permiso para el trabajador para el nacimiento de su hijo que se suman a la disposición de la Ley n. 92/2012 (Ley Fornero); licencia remunerada de 10 días por hospitalización de niños menores de 12 años; provisión de cursos de actualización para la trabajadora que se reincorpora al trabajo tras el disfrute del permiso de maternidad; uso de remote working (en la perspectiva, se supone, del trabajo ágil) parte de días en el mes (ej. 10 días.) o de la semana (es. 3 días)

9. Entre estos contratos hay un acuerdo de proximidad (Sangiusto Ltd., enero de 2015), que contiene numerosas disposiciones de excepción: en tema de movilidad interna prevé un periodo de 365 días al año o 400 en los 24 meses antes de producir los efectos de la promoción a nive profesional superior y el acceso a los contratos a tiempo determinado hasta el $100 \%$ de orgánico.
Sin embargo, no se oculta el pesar por el reducido número de acuerdos que tratan de conciliación y welfare para el año 2015 - aunque el proceso de recopilación no se ha completado - lo que sugiere un cambio de tendencia conectada de manera extraña a la leve recuperación económica en los últimos meses.

Al menos por lo que surge de los acuerdos estudiados desde el comienzo de 2015, el interés de las partes se centró en la revitalización de la producción, evitando completamente una visión más amplia del bienestar en el trabajo que sin duda afecta a la performance de los trabajadores.

La función de mejorar las condiciones de trabajo asignado a la negociación colectiva, que ya en las últimas décadas se ha reducido en el ámbito nacional, parece haber sufrido con la crisis más reciente un aplazamiento adicional tambien a nivel descentralizado (desde una perspectiva europea: Euround, 2014); niveles que deberían ser capaces de entender las necesidades del territorio local y de las empresas que existen en el mismo territorio.

Esto no quiere decir que en la Provincia de Brescia no se experimente la excelencia, en términos de herramientas de welfare y conciliación para el personal desde la perspectiva del bienestar organizacional como resultado de una visión comprometida de algunos empleadores, pero actualmente carece de un intercambio de este tipo de experiencias.

El trabajo de recopilación y análisis de la situación de la negociación en nuestra área es sólo uno de los objetivos del Observatorio. Un segundo objetivo, a más largo plazo, se sitúa en meter "en red" tales experiencias como el primer paso para la construcción de los modelos locales en el que la mimesis y el aprendizaje mutuo permitirán la circulación de as mejores soluciones. 


\section{Bibliografía básica.}

Aa.Vv. (2015), Il sistema retributivo premiale in Fiat-Chrysler Automobile (FCA). Elementi d un nuovo modello contrattuale?, in DLRI, n. 148, p. 651 ss

Aa.Vv. (2011), Il caso Fiat: una crisi di sistema?, en $L D$, numero monografico.

Ackers, Peter (2016), Free collective bargaining and incomes policy: learning from Barbara Wootton and Hugh Clegg on post-war British Industrial Relations and wage inequality, en IRJ, n. 47 , p. 434 ss.

Baccaro, Lucio; Howell, Chris (2011), A Common Neoliberal Trajectory: The Transformation of Industrial Relations in Advanced Capitalism, en Politics \& Society, p. 521 ss.

Barbera, Marzia; Protopapa, Venera (2014), Il caso Fiat: come la tutela antidiscriminatoria riformula il conflitto sindacale, en $R G L$, n. 1, p. 163 ss.

Berta, Giuseppe (2014), Produzione intelligente. Un viaggio nelle nuove fabbriche, Torino, Einaudi.

Bologna, Silvio (2016), Liberalizing Industrial Relations in Southern-Europe: Towards the End of a Coordinated and Egalitarian Model, en Economia \& Lavoro, n. 2, p. 101 ss.

Campanella, Piera (2013), Decentramento contrattuale e incentivi retributivi nel quadro delle politiche di sostegno alla produttività del lavoro, en WP D'Antona, n. 185.

Carinci, Franco (2014) (a cura di), II Testo unico sulla rappresentanza 10 gennaio 2014 , Adapt University Press.

Carrieri, Mimmo; Treu, Tiziano (2013), Introduzione. Le relazioni industriali italiane ed europee: innovazioni da completare e convergenze da affinare, Bologna, II Mulino, p. 7 ss.

Cella, Gian Primo (2016), Quale futuro per la contrattazione collettiva, in $D L R I$, n. 150, p. 217 ss

Cella, Gian Primo (2013), Una sentenza sulla rappresentanza sindacale (o del lavoro?), en $L D$, p. 509 ss.

D’Arcangelo, Lucia (2012), Contrattazione territoriale e sviluppo locale, Torino, Giappichelli.

De Felice, Alfonsina (2015), Potere locale e metodo concertativo fra i due secoli. Cronaca di una bruciante illusione, en RGL, n. 1, p. 79 ss.

Del Punta, Riccardo (2013), L'art. 19 Statuto dei lavoratori davanti alla Consulta: una pronuncia condivisibile ma interlocutoria, en $L D$, p. 527 ss.

Di Nardo, Filippo (2015), Il futuro del welfare è in azienda, Milano, Guerini.

Eurofound (2014), Impact of the crisis on industrial relations and working conditions in Europe, Dublin.

Guarriello, Fausta (2016), Crisi economica, contrattazione collettiva e ruolo della legge, in $D L R I$, n. 149, p. 3 ss
Ichino, Pietro (2005), A che cosa serve il sindacato?, Milano, Mondadori.

Ires (2015), La contrattazione sociale negli anni della crisi. Sesto rapporto sulla contrattazione sociale territoriale, en $h$ ttp://www.ires.it/

Keune, Maarten (2011), Decentralizing Wage Setting in the Time of Crisis, en ELLJ, p. 86 ss. Leccese, Vito (2013), Partecipazione alle trattative, tutela del dissenso e art. 19 dello Statuto dei lavoratori, en $L D$, p. 539 ss.

Liso, Franco; Corazza, Luisa (2015), Le clausole di pace: variazioni sul tema, en WP D’Antona n. 247.

Maino, Franca; Ferrera, Maurizio (2013) (a cura di), Primo rapporto sul secondo welfare in Italia 2013, Torino, Centro di documentazione e ricerca Luigi Einaudi.

Napoli, Mario (2014), Rsa e Costituzione. La giurisprudenza della Corte Costituzionale, Milano, Vita e Pensiero.

Rebaudengo, Paolo (2015), Nuove regole in fabbrica. Dal contrato Fiat alle nuove relazioni industriali, Bologna, II Mulino.

Regalia, Ida (2015a), Negoziare sul territorio. Un'introduzione, en RGL, n. 1, p. 3 ss.

Regalia, Ida (2015b), Oltre la contrattazione di secondo livello. Note sulla concertazione a livello locale, en RGL, n. 1, p. 97 ss.

Ricciardi, Mario (2014), La parabola. Ascesa e declino della contrattazione collettiva in Italia Bologna, Clueb.

Streeck, Wolfgang (2009), Re-Forming Capitalism, Oxford University Press, Oxford.

Tomassetti, Paolo (2015), Relazioni industriali e risorse umane. Sindacato e contrattazione aziendale concessiva: una ricerca empirica, en $D R I$, n. 1, p. 183 ss.

Vaughan-Whitehead, Daniel (2015) (ed), The European Social Model in Crisis: Is Europe Losing Its Soul?, ILO (Elgar), Geneva.

Visser, Jelle (2013), ICTWSS, Database on Institutional Characteristics of Trade Unions, Wage-settings, State Intervention and Social Pacts in 34 Countries between 1960 and 2012, April 2013, Amsterdam Institute for Advanced Labour Studies (AIAS), University of Amsterdam.

Zoppoli, Lorenzo (2015), Istituzioni e negoziazioni territoriali: un'analisi della strumentazione giuridica, en $R G L$, n. 1 , p. 29 ss. 


\section{Abreviaturas.}

DLRI Giornale di diritto del lavoro e di relazioni industriali

DRI Diritto delle relazioni industriali

ELLJ European Labour Law Journal

IRJ Industrial Relations Journal

LD Lavoro e diritto

RGL Rivista giuridica del lavoro

WP D'Antona Working Paper Centro Studi D'Antona 